Rapid Reviews COVID-19

\title{
Review 2: "Integrated \\ Single-Cell Atlases Reveal \\ an Oral SARS-CoV-2 \\ Infection and Transmission \\ Axis"
}

Ophir Klein ${ }^{\mathbf{1}}$, Irit Miller Zmora

${ }^{1}$ UCSF, Orofacial Sciences

Published on: Jan 18, 2021

DOI: $10.1162 / 2 \mathrm{e} 3983 f 5.692 \mathrm{ad} 3 \mathrm{~d} 7$

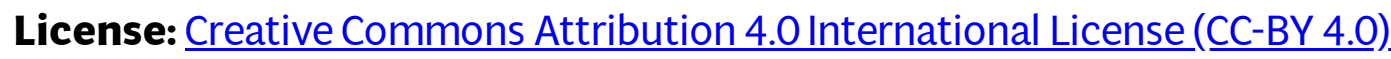




\section{$\underline{\text { RR:C19 Evidence Scale rating by reviewer: }}$}

- Strong. The main study claims are very well-justified by the data and analytic methods used. There is little room for doubt that the study produced has very similar results and conclusions as compared with the hypothetical ideal study. The study's main claims should be considered conclusive and actionable without reservation.

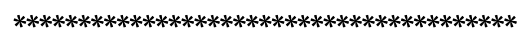

\section{Review:}

Using single-cell RNA sequencing (scRNAseq), the authors set out to study the oral cavity as a site of SARS-CoV-2 infection. Huang et al. generated an atlas of cell types from different regions of the oral cavity. They characterized the expression of genes that enable viral entry and infection, and they examined antibodies that suggest the possibility of local oral infection. Overall, the manuscript focuses on important questions and will lay a foundation for future examination of the role of the oral cavity in COVID-19.

While COVID-19 is known to result in a variety of oral symptoms such as dry mouth, blisters on the lining mucosa, and ageusia, the majority of reports to date have focused on the respiratory system. This study is primarily aimed at characterizing the cellular susceptibility of diverse oral epithelia to infection and the potential role of these tissues in viral transmission. The authors established a comprehensive human oral cell atlas, based on scRNAseq, thereby enabling quantification of cell and niche-specific susceptibility to oral SARS-CoV-2 infection. They identified 50 cell types in the gingiva, hard palate mucosae, and salivary glands (SG). Through computational analyses, the manuscript characterizes the different cell subpopulations, including various immune cells, and defines the specific expression of viral entry factor genes. The analysis reveals multiple epithelial subpopulations that express ACE2 and TMPRSS2, supporting the hypothesis that oral epithelia are susceptible to SARS-CoV-2 infection. To further analyze the oral sites that were not represented within the human samples, an integrated human and mouse atlas was generated, revealing a moderate correlation between human and mouse cell clusters. Similar to human data, in the mouse Ace 2 and Tmprss 2 are expressed in SG and differentiated tongue epithelia. These findings were further validated using in situ hybridization across oral sites, mainly the SG ducts and acini, as well as the suprabasal layer of the mucosal epithelium. Autopsies from patients with COVID-19 indicated viral RNA and inflammatory response in the presence of the virus in the SG. Identification of the shedding of stratified squamous cells that express the viral entry factors and viral RNA may, in parallel to an increase in epithelial cell shedding, suggest an effective response resulting in increased shedding of infected cells. However, further analysis will be required in the future to establish the specific mucosal response to infected 
cells. The authors alternatively suggest that shed epithelium could provide a potential route for viral spread. Similarly, this aspect will need future clarification by isolating these cells and monitoring viral replication and the ability to infect other cells in in vitro culture. Characterization of viral load in the saliva of several subjects showed presence of virus after nasal swabs were negative, suggesting sustained viral shedding from oral epithelium. These data point to the need for further assessment of viral viability in and replicative ability of the shed cells. The authors also found anti-SARS-CoV-2 antibodies in the saliva, further indicating a local infection of the oral cavity.

To summarize, this study provides a thorough analysis of the susceptibility of the SG and oral mucosa to SARS-CoV-2 infection. These findings support the use of masks and social distancing to prevent transmission. Future research is needed to establish the pathways by which viral infection results in oral related symptoms. The claims are very well-supported by the data and methods used, and decision-makers should consider the claims in this study actionable without reservation based on the methods and data. 